\title{
Shear effects on passive scalar spectra
}

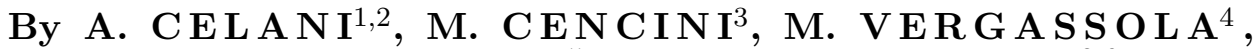 \\ E. VILLERMAU X A A D D. VINCE N Z I ${ }^{2,6}$ \\ ${ }^{1}$ CNRS, INLN, 1361 Route des Lucioles, F-06560 Valbonne, France. \\ 2 CNRS UMR 6202, Observatoire de la Côte d'Azur, B.P. 4229, 06304 Nice Cedex 4, France. \\ ${ }^{3}$ Center for Statistical Mechanics and Complexity, INFM Roma 1 and Dipartimento di Fisica, \\ Università di Roma "La Sapienza", Piazzale Aldo Moro, 2, I-00185 Roma, Italy. \\ ${ }^{4}$ CNRS, URA 2171, Institut Pasteur, 28, rue du Dr. Roux, 75724 Paris Cedex 15, France. \\ ${ }^{5}$ Univ. Aix Marseille 1, IRPHE, 49, rue Frederic Joliot Curie, F-13384 Marseille 13, France. \\ ${ }^{6}$ Dep. de Mathématiques, Univ. de Nice-Sophia Antipolis, Parc Valrose, 06108 Nice, France.
}

(Received 5 July 2021)

The effects of a large-scale shear on the energy spectrum of a passively advected scalar field are investigated. The shear is superimposed on a turbulent isotropic flow, yielding an Obukhov-Corrsin $k^{-5 / 3}$ scalar spectrum at small scales. Shear effects appear at large scales, where a different, anisotropic behavior is observed. The scalar spectrum is shown to behave as $k^{-4 / 3}$ for a shear fixed in intensity and direction. For other types of shear characteristics, the slope is generally intermediate between the $-5 / 3$ Obukhov-Corrsin's and the -1 Batchelor's values. The physical mechanisms at the origin of this behaviour are illustrated in terms of the motion of Lagrangian particles. They provide an explanation to the scalar spectra shallow and dependent on the experimental conditions observed in shear flows at moderate Reynolds numbers.

\section{Introduction}

Two major regimes are known for a passive scalar field transported by a turbulent flow. The first, known as inertial-convective, refers to the range of scales where the direct effects of the fluid viscosity and the scalar diffusivity are both negligible. The predicted behaviour of the spectrum $E_{\theta}(k)$ of the scalar field $\theta$ is (Obukhov (1949), Corrsin (1951)):

$$
E_{\theta}(k)=C \epsilon^{-1 / 3} \epsilon_{\theta} k^{-5 / 3} .
$$

Here, $k$ denotes the wavenumbers, $C$ is an adimensional constant, $\epsilon$ and $\epsilon_{\theta}$ are the average dissipation rates of the kinetic energy and the scalar variance, respectively. The second regime, dubbed viscous-convective, is at scales smaller than the former and holds for weakly diffusive (alternatively high Schmidt, or Prandtl number) scalars. The velocity field is now smoothed out by the molecular viscosity, linearizing the dependence of the velocity increments on the separation between the two points where the increment is taken. That yields the Batchelor behaviour (Batchelor (1959)):

$$
E_{\theta}(k) \propto k^{-1} .
$$

Scalar spectra have been measured in a number of laboratory experiments. None of these pure power laws with the above anticipated exponents are routinely observed. The existence of a $k^{-1}$ in the viscous-convective subrange has been questioned based on high Reynolds and Schmidt numbers experiments (Miller \& Dimotakis (1996), Warhaft (2000) 
Yeung, Xu \& Sreenivasan (2002)). As for larger scales, scalar spectra do behave compatibly with a power law, but their slope is often found to deviate from the ObukhovCorrsin $5 / 3$ value. Deviations are particularly important in turbulent shear flows, where the observations indicate that the spectra strongly depend on the Reynolds number $R e$ (Mestayer (1982), Sreenivasan (1991), Sreenivasan (1996), Miller \& Dimotakis (1996) Warhaft (2000) and on the detailed set-up of the advecting turbulent flow. The prediction (1.1) is in particular recovered for very large Reynolds numbers only $\left(R e_{\lambda}>2000\right)$. Deviations are observed also when the velocity field displays a convincing $k^{-5 / 3}$ spectrum (see, e.g., Sreenivasan (1996), Villermaux, Innocenti \& Duplat (2001)).

Our aim here is to investigate the physical origin of the behaviour experimentally observed in shear flows, briefly recalled in $\S 2$. We specifically consider a model where the velocity is decomposed into a linear shear, superimposed onto a turbulent flow supposed to obey Kolmogorov's scaling. As in thermal convection (see, e.g., Siggia (1994)), the presence of the shear introduces a new length-scale $r_{c}$. The scalar spectrum crosses from the classical behavior (1.1) at scales $r \ll r_{c}$ to a different anisotropic behavior for $r \gg r_{c}$. As discussed in $\S 3$, Lagrangian dimensional arguments allow to predict the scaling of the scalar spectrum in the new shear-regime for a variety of shear properties. A $-4 / 3$ scaling is, in particular, found for a shear fixed in intensity and direction. A test of the Lagrangian dimensional arguments is provided in $\S 4$, where we consider a simpler turbulent flow with a short correlation time. The assumption is unphysical and the very same Lagrangian arguments of $\S 3$ yield quantitatively different predictions; the point is however that these predictions can be stringently tested against the exact solution for the scalar spectrum which can be obtained in this special case. The final Section is devoted to discussions and conclusions. The Appendix is reserved to technical material used in $\S 4$.

\section{Scalar spectra in experiments}

Turbulent flows might be divided into two broad categories: shear flows (jets, wakes, and boundary layers) and flows where the effects of large-scale shears are tamed (e.g., grid-generated turbulence). The latter are the laboratory flows providing the closest physical realization of the homogeneous and isotropic turbulence which most of the theoretical fluid dynamics deals with.

Various possible ways to inject scalar fields in turbulent flows have been employed. In grid turbulence, temperature fluctuations are generated by heating either the grid itself or small wires located thereafter. In shear flows, one possibility is to weakly heat the jet or the wall of the boundary layer. Alternatively, a colorant dye can be injected past the flow source, at a scale possibly different from that of the stirring source. Temperature time series are taken by a thermometer in a fixed location and, as customary in most fluid dynamics experiments, time distances are converted to space distances by Taylor hypothesis. Scalar spectra or, equivalently, second-order structure functions, are thus measured. The use of fluorescent dyes offers the possibility to extract cuts -usually two-dimensional- of the scalar field from the intensity field. Simultaneous velocity measurements are usually taken by anemometers. The reader interested in a detailed discussion of the experimental techniques is referred to Sreenivasan (1996), Warhaft (2000) Villermaux, Innocenti \& Duplat (2001).

The observations gathered in the literature might be summarized as follows. In gridgenerated turbulence, the prediction (1.1) is well verified. That holds also at moderate $R e_{\lambda}$, when there is just a tiny range of scales where the spectrum of the velocity field displays a clean $-5 / 3$ slope. Some results obtained heating directly the grid used to 
generate the turbulence have led to some criticisms (Warhaft (2000) on account of possible correlations between the temperature and the velocity. It seems, nevertheless, a well-established fact (see Jayesh, Tong \& Warhaft (1994), Mydlarski \& Warhaft (1998) Warhaft (2000) that the slope of the scalar spectrum increases with $R e_{\lambda}$ from values $\simeq 1.5$ to $5 / 3$, reached at Reynolds numbers $R e_{\lambda} \geq 200$. Small deviations observed at lower $R e_{\lambda}$ are confidently ascribed to finite $R e$-number effects.

The situation in turbulent shear flows is more intricate. Experiments with different $R e_{\lambda}$ 's but similar non-dimensional shear rates have been collected and compared by Sreenivasan (1996) The main observation is that the slopes of the passive scalar spectra display a strong dependence on the turbulent intensity. Their slope is approximately 1.3 at low $R e_{\lambda}$ and attains the predicted $5 / 3$ value at $R e_{\lambda} \geq 2000$ only. Strong anisotropic effects are also observed for the velocity energy spectrum. Indeed, its longitudinal component reaches the expected $-5 / 3$ behaviour at moderate $\operatorname{Re}_{\lambda}(\approx 50)$. Conversely, its transverse component behaves more similarly to the scalar field, with a slope continuously increasing with $R e_{\lambda}$ and saturating to $5 / 3$ only for $R e_{\lambda} \geq 3000$. The experiments by Villermaux, Innocenti \& Duplat (2001) report even more severe deviations from (1.1), with a slope close to the Batchelor unit value, in spite of the $-5 / 3$ spectrum observed for the velocity field. The scalar injection scale was, although still lying in the inertial range of scales, smaller than the turbulence forcing scale in that case.

\section{Shear effects on Lagrangian dynamics}

The experimental observations presented in the previous Section naturally lead to surmise that the deviations observed in the scalar spectra have, among other possibles causes, their origin in the presence of a sustained large-scale shear. The interplay between diffusion and shear is a classical problem first examined by Levèque (1928) for heat transport across boundary layers. Qualitatively, the flow stretches the distances between particles along the shear and sharpens the scalar gradient perpendicular to it, therefore altering the usual diffusion law.

In order to quantitatively assess the impact of shear on scalar spectra, we introduce the following simple model for the velocity field $\boldsymbol{v}(\boldsymbol{r}, t)$ :

$$
\boldsymbol{v}(\boldsymbol{r}, t)=\sigma y \hat{\boldsymbol{x}}+\boldsymbol{u}(\boldsymbol{r}, t) .
$$

The flow is thus the superposition of an average linear shear of intensity $\sigma$ directed along the $x$-direction and a turbulent fluctuating field $\boldsymbol{u}$, supposed to obey Kolmogorov's scaling, for the sake of simplicity. The spectrum of the velocity field obeys in particular the classical $k^{-5 / 3}$ law.

A passive scalar field, $\theta(\boldsymbol{r}, t)$, transported by the above flow is governed by the standard advection-diffusion equation:

$$
\partial_{t} \theta+\sigma y \partial_{x} \theta+\boldsymbol{u} \cdot \boldsymbol{\nabla} \theta=\kappa \Delta \theta+f,
$$

where $\kappa$ is the molecular diffusivity and $f$ is an external source of scalar fluctuations. The source is needed to maintain the system in a statistically steady state and we shall suppose that the forcing has a characteristic length $L_{f}$.

The transport equation (3.2) is equivalently recast in terms of the Lagrangian trajectories $\boldsymbol{\rho}(t)$ of tracer particles. They obey the stochastic differential equation

$$
d \boldsymbol{\rho}=\boldsymbol{v}(\boldsymbol{\rho}, t) d t+\sqrt{2 \kappa} d \boldsymbol{W},
$$

where $\boldsymbol{W}$ is an isotropic Brownian motion and $\boldsymbol{v}$ is given by (3.1). The equation for the scalar field along the trajectories is the ordinary differential equation $d \theta / d t=f$, 
easily integrated. The scalar second-order correlation function of the scalar field is then expressed as (see, e.g., Falkovich, Gawędzki \& Vergassola (2001)):

$$
C_{2}(\boldsymbol{r}, t)=\langle\theta(\boldsymbol{r}, t) \theta(\mathbf{0}, t)\rangle=\int_{-\infty}^{t} \mathrm{~d} s \int \mathrm{d} \boldsymbol{R} p(\boldsymbol{R}, s \mid \boldsymbol{r}, t) \chi(\boldsymbol{R}) .
$$

Here, $\boldsymbol{R}$ is the separation between two tracer particles and $p(\boldsymbol{R}, s \mid \boldsymbol{r}, t)$ is the propagator, i.e. the probability, averaged over the realizations of the velocity ensemble and of the Brownian noise, that a pair of particles is found separated by $\boldsymbol{R}$ at time $s$, conditional to its separation $\boldsymbol{r}$ at time $t$. The function $\chi(\boldsymbol{R})$ having its support at scales $R \leq L_{f}$, the integral (3.4) is roughly equal to $\chi(0) T\left(r, L_{f}\right)$, where $T\left(r, L_{f}\right)$ is the residence time at distances $\leq L_{f}$ for a pair of particles initially separated by a distance $r$. Analogously, the second-order structure function $S_{2}(r)=2\left[C_{2}(0)-C_{2}(r)\right] \propto T\left(0, L_{f}\right)-T\left(r, L_{f}\right)$. The incompressibility of the flow ensures that the scaling of the latter quantity can be estimated as the time for two particles, initially coinciding, to reach a separation $r$.

The problem of determining the scaling behaviour of scalar spectra is thus reduced to the study of the evolution of the separation between a pair of particles.

For the case without shear, $\sigma=0$, classical dimensional arguments give the Richardson law: $\left\langle R^{2}(t)\right\rangle \sim t^{3}$, which translates into a $2 / 3$ scaling exponent for $S_{2}(r)$ and the ensuing Obukhov-Corrsin $-5 / 3$ slope for the scalar spectrum.

Let us now consider the case with shear, $\sigma \neq 0$. The components of the separation between two fluid particles obey the equations of motion:

$$
\begin{aligned}
\dot{R}_{x} & =\sigma R_{y}+\delta u_{x}(\boldsymbol{R}, t), \\
\dot{\boldsymbol{R}}_{\perp} & =\delta \boldsymbol{u}_{\perp}(\boldsymbol{R}, t),
\end{aligned}
$$

where the dot indicates the time-derivative, $\delta \boldsymbol{u}$ is the velocity difference between the two particles and $\dot{\boldsymbol{R}}_{\perp}$ indicates the components of the velocity orthogonal to the shear. At small enough times, the turbulent component, which scales with exponent $1 / 3$, dominates over the linear shear and Richardson's behaviour holds. The crossover to a different anisotropic behaviour occurs at $r_{c} \simeq \sigma^{-3 / 2}$. Those are the separations where the shear and the turbulent components become comparable. The time required for two particles initially coinciding to reach those scales behaves as $t_{c} \propto \sigma^{-1}$. For $t \gg t_{c}$, the form of the Lagrangian equations naturally suggests that the parallel and the transverse components scale differently with time:

$$
\left\langle R_{x}^{2}\right\rangle \approx\left\langle R^{2}\right\rangle \propto t^{2 \alpha}, \quad \text { and } \quad\left\langle R_{\perp}^{2}\right\rangle \sim t^{2 \beta} .
$$

Inserting this ansatz into (3.5) and (3.6), neglecting the $\delta u_{x}$ term in (3.5) and using the Kolmogorov's scaling $\delta \boldsymbol{u}_{\perp}(\boldsymbol{R}, t) \propto R^{1 / 3} \simeq t^{\alpha / 3}$, we obtain the relations: $\alpha-1=\beta$ and $\beta-1=\alpha / 3$. We thus end up with the predictions $\alpha=3, \beta=2$ for the Lagrangian separations, that give

$$
C_{2}(r) \propto r^{1 / 3} ; \quad E(k) \propto k^{-4 / 3},
$$

for the correlations of the scalar field, by using the arguments following (3.4).

It is worth emphasizing that the scalar statistics is sensitive to the shear characteristics, as it should be expected for any passive transport. For example, if the direction of the shear were to rotate rapidly, isotropy would be recovered and the stretching of the separations among the particles would be dominated by the linear shear component, leading to an exponential-in-time separation and a $k^{-1}$ behaviour. The physics is the same as in the Batchelor regime, but the behaviour now holds at large scales. The rapidity of the rotation should be gauged with respect to the Lagrangian turn-over time: if $\tau_{\phi}$ denotes the typical time of rotation, the fixed limit (3.1) holds for scales $r \ll \tau_{\phi}^{3 / 2}$ and 
the rapidly rotating asymptotics in the opposite limit. As for the fluctuations of the shear intensity, let us similarly denote by $\tau_{\sigma}$ its correlation time. The intensity is effectively fixed in time, as in (3.1), for scales $r \ll \tau_{\sigma}^{3 / 2}$. In the opposite limit, the intensity might be taken as a random process with a short correlation time $\left\langle\sigma(t) \sigma\left(t^{\prime}\right)\right\rangle=\sigma^{2} \delta\left(t-t^{\prime}\right)$. Inserting the ansatz (3.7) into (3.5) leads now to the relation $\alpha-1=-1 / 2+\beta$, giving a slope $-13 / 9$ for the scalar spectrum. The geometry of the shear and the ratio between its time-scale and the turbulent turn-over times are thus seen to affect the properties of a passively transported field. The point of interest to interpret the experimental data is that the exponents of the spectra are in all cases smaller than $5 / 3$, implying that the presence of a shear generally induces a crossover to a shallower spectrum at the large scales.

\section{A solvable case}

The aim of this Section is to test the dimensional arguments presented in the previous section by investigating the simpler case of a turbulent flow $\boldsymbol{u}$ belonging to the Kraichnan ensemble (Kraichnan (1968)). The rationale is as follows. The short correlation time of the velocity is unphysical and the Lagrangian predictions (3.8) are modified thereby (the scaling $-4 / 3$ of the scalar spectrum becoming, for example, $-11 / 9$ ). Yet, the point is that one can compare those predictions versus the solution of the exact equation for the scalar spectrum. The advantage over a direct numerical simulation of the more realistic flows in Section 3 is that the range of scales available here is much more extended and the comparison is therefore quantitatively more stringent, albeit less direct.

The field $\boldsymbol{u}$ in (3.1) is taken now as an incompressible, statistically isotropic and homogeneous Gaussian field of zero mean and correlation function:

$$
\left\langle\left[u_{i}(\boldsymbol{r}, t)-u_{i}(\mathbf{0}, t)\right]\left[u_{j}(\boldsymbol{r}, 0)-u_{j}(\mathbf{0}, 0)\right]\right\rangle=\delta(t)\left\{D r^{\xi}\left[(1+\xi) \delta_{i j}-\xi \frac{r_{i} r_{j}}{r^{2}}\right]\right\} .
$$

Here, we specialize to the two-dimensional case for the sake of simplicity: this choice bears no qualitative consequences on the results. The parameter $D$ measures the turbulent intensity and the velocity field is assumed to be scale-invariant with roughness exponent $\xi \in[0,2]$. The presence of an ultraviolet viscous cutoff mimicking the Kolmogorov scale might be considered, but it will not be needed here.

Using standard methods of Gaussian integration by parts (see Falkovich, Gawędzki \& Vergassola (2001)), we can re-adapt the Lagrangian arguments of the previous section to the case of a Kraichnan flow. The Lagrangian separation law for the isotropic case is: $\left\langle R^{2}(t)\right\rangle \propto t^{2 /(2-\xi)}$. Note that the Richardson scaling is obtained for $\xi=4 / 3$, differing from the $2 / 3$ of real turbulent flows due to the $\delta$-correlation in time of the velocity field. For the anisotropic case of a shear fixed in intensity and direction, the relations between the exponents $\alpha$ and $\beta$ stemming from (3.5) and (3.6) read now: $\alpha-1=\beta$ and $2 \beta-1=2 \alpha \xi$. Solving these relations gives the following behaviors of the Lagrangian separations and the scalar spectra:

$$
\left\langle R_{x}^{2}\right\rangle \approx\left\langle R^{2}\right\rangle \propto t^{6 /(2-\xi)} ; \quad\left\langle R_{y}^{2}\right\rangle \sim t^{2(1+\xi) /(2-\xi)} ; \quad E(k) \propto k^{-(5-\xi) / 3} .
$$

For $\xi=4 / 3$, (4.2) gives a $-11 / 9$ exponent for the scaling of the scalar spectrum.

The advantage of Kraichnan's flows is that the behavior of the second-order correlation function $C_{2}(r)$ at the stationary state can be determined exactly and used to test the previous dimensional arguments. In the shear-free case, $\sigma=0$, the problem is isotropic and the expression of $C_{2}(r)$ is well-known analytically. The inertial-range scaling be-

haviour for the second-order structure function $S_{2}(r)=\frac{\chi(0)}{(2-\xi) D} r^{2-\xi}$, where $\chi(0)$ is the 


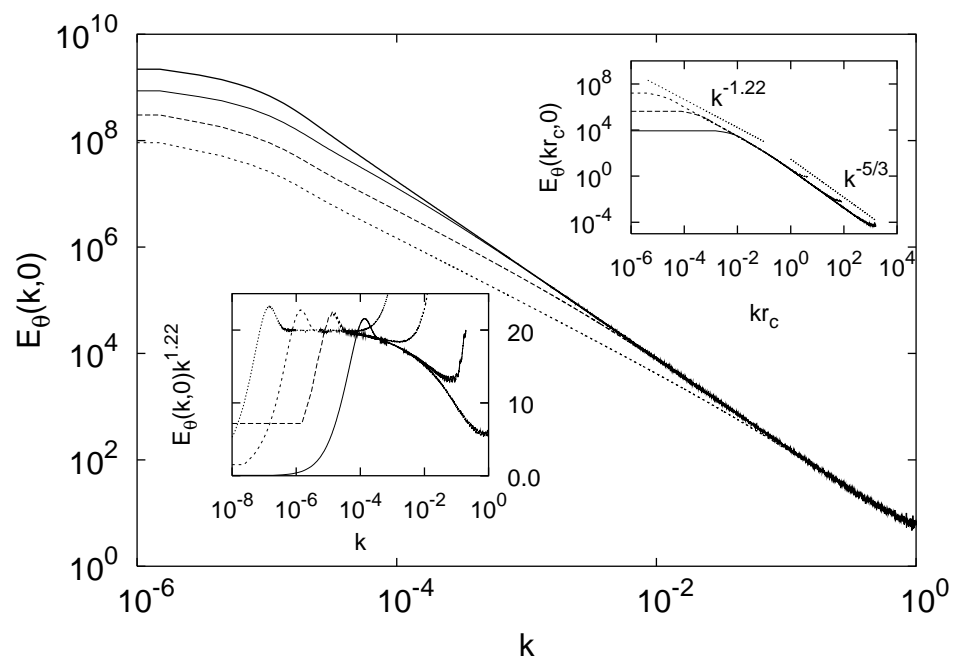

FiguRE 1. Scalar spectra in the $x$-direction, $E_{\theta}(k, 0)$, obtained by fixing $D=0.05$ and varying $\sigma$ (from top to bottom, $\sigma=0,0.0004,0.0036,0.025$ ). In the numerical computations $L_{b o x}$ and $L_{f}$ have been fixed to $128^{3}$ and $10^{5}$, respectively - see the Appendix for the definition of the parameters - and the scaling exponent of the velocity $\xi=4 / 3$. The inset on the top right shows the rescaled spectra $E_{\theta}\left(k r_{c}, 0\right) v s k r_{c}$. At large wavenumbers, the $k^{-5 / 3}$ spectrum is recovered, while at small $k$ the power law $k^{-11 / 9}$, predicted by Lagrangian dimensional arguments, appears. The inset on the bottom left shows the compensated spectra $E_{\theta}(k, 0) k^{11 / 9}$ obtained by fixing $\sigma=0.025$ and varying $L_{f}=10^{4}, 10^{5}, 10^{6}, 10^{7}$. The number of harmonics $\ell^{*}$, the box size $L_{b o x}$ and the number of grid points have been varied accordingly. Note that the transition region to the asymptotic slope $-11 / 9$ is quite broad.

scalar variance injection rate. The exponent $2-\xi$ is in agreement with the Lagrangian dimensional estimate.

In the presence of shear, the equation for the second-order correlation function is still closed, but the problem is not isotropic anymore. Following the same procedure as in the shear-free case (Kraichnan (1968)), one obtains:

$$
\begin{aligned}
\partial_{t} C_{2} & +\sigma\left(r \sin \phi \cos \phi \partial_{r}-\sin ^{2} \phi \partial_{\phi}\right) C_{2}=\frac{1}{r} \partial_{r}\left(D r^{1+\xi}+2 \kappa r\right) \partial_{r} C_{2} \\
& +\frac{1}{r^{2}}\left[(1+\xi) D r^{\xi}+2 \kappa\right] \partial_{\phi}^{2} C_{2}+\chi(r)
\end{aligned}
$$

where $\boldsymbol{r}=(r, \phi)$ denotes the relative distance between the two points. The forcing is taken Gaussian, statistically homogeneous and isotropic, with zero mean and correlation function $\langle f(\boldsymbol{r}, t) f(0,0)\rangle=\delta(t) \chi(r)$. In the simulations, the correlation function of the forcing is taken as $\chi(r)=\exp \left(-r^{2} / 2 L_{f}^{2}\right)$. Contrary to the $\delta$-correlation of the velocity, the previous hypotheses on the forcing are not restrictive and might be easily relaxed. The anisotropy induced by the shear makes that $C_{2}$ will depend both on $r$ and on the angle $\phi$ between $\boldsymbol{r}$ and the $x$-axis. The steady-state solution of Eq. (4.3) cannot be obtained analytically anymore and we have to resort to numerical methods (see the Appendix for details).

The existence of a crossover scale, $r_{c}$, separating the scales dominated by the turbulence and those affected by the shear, is easily recognized by a direct inspection of Eq. (4.3). A simple balance of the shear and the eddy-diffusivity terms in Eq. (4.3) gives: $r_{c} \sim\left(\frac{D}{\sigma}\right)^{\frac{1}{2-\xi}}$. The crossover is well evident in Fig. 1, where the scalar spectrum for $\xi=4 / 3$ in a wide 

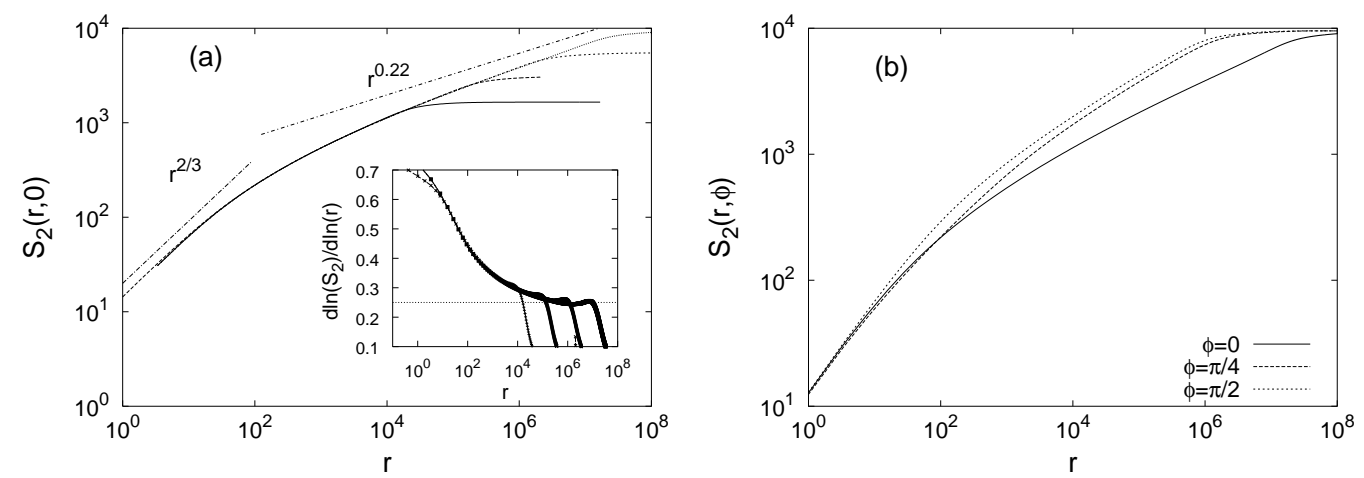

Figure 2. (a) The scalar second-order structure function measured in the $x$-direction, $S_{2}(r, 0)$, fixing $\sigma$ and varying $L_{f}$ (as in the bottom inset of Fig. 1). The inset shows the local slopes. Note that the approach to the asymptotic scaling $r^{0.22}$ is even slower than in the spectra. (b) The scalar second-order structure function measured at different angles, $S_{2}(r, \phi)$, for the case $\sigma=0.025$ and $L_{f}=10^{7}$. The statistics becomes more and more anisotropic as larger scales are considered. A similar behaviour is observed for the spectra (not shown).

span of scales is reported. The results are in agreement with the Lagrangian predictions (4.2), with $E_{\theta}(k) \sim k^{-5 / 3}$, for $k r_{c} \gg 1$, and $E_{\theta}(k) \sim k^{-11 / 9}$ asymptotically reached at the large scales. Moreover, as shown in the top right inset, the spectra obtained with different shear rates all collapse by the rescaling of the wavenumbers $k r_{c}$.

The corresponding behaviour in physical space for the second-order structure function is shown in Fig. 2a. Two regimes are again clearly visible. A point to be remarked in the inset is that the convergence to the asymptotic slope, $S_{2}(r) \sim r^{2 / 9}$, is even slower than in $k$-space. The behaviour of the second-order structure function for different orientations is shown in Fig. 2b. As expected in a range of scales affected by the shear, the anisotropy is strong and the crossover between the two previous regimes depends on the angle of measurement. It would be very informative to have similar measurements of the anisotropy in experimental conditions.

Note, as a side remark, that an asymptotic expansion valid for small angles can be found analytically. Indeed, the correlation function $C_{2}$ can be expressed as

$$
C_{2}\left(r_{x}, r_{y}\right)=(D / \sigma)^{-1 / 3} r_{x}^{(2-\xi) / 3} f\left[r_{y} /\left(D r_{x}^{1+\xi} / \sigma\right)^{1 / 3}\right],
$$

where the angular part $f$ obeys the equation:

$$
f^{\prime \prime}(w)+\frac{w^{2}}{3} f^{\prime}(w)-\frac{w}{(1+\xi)} f(w)=-\frac{\chi}{\sigma(1+\xi)} .
$$

For small angles, i.e. $w \ll 1$, the operator appearing in (4.3) reduces to $\sigma r_{y} \partial_{r_{x}}-D(1+$ $\xi) r_{x}^{\xi} \partial_{r_{y}}^{2}$ and it is easy to check that the solution to 4.5] has the form (4.4) with the function $f$ behaving as $f(w)=-1-\frac{\chi w^{2}}{2 \sigma(1+\xi)}-\frac{w^{3}}{6(1+\xi)}+O\left(w^{4}\right)$.

An even closer contact to the Lagrangian arguments (4.2) is made by directly simulating the equations of motion for the tracer particles. The method used for the simulations is the same as in Frisch et al. (1998) Fig. 3 presents the evolution of the particle separation for $\xi=4 / 3$. As long as $\left\langle R^{2}(t)\right\rangle<r_{c}^{2} \sim\left(\frac{D}{\sigma}\right)^{\frac{2}{2-\xi}}$, the Richardson prediction is recovered and the anisotropy induced by the shear is negligible. As the separation becomes larger and larger compared to $r_{c}$, the trajectories are more and more affected by the shear and $\left\langle R^{2}\right\rangle \approx\left\langle R_{x}^{2}\right\rangle \gg\left\langle R_{y}^{2}\right\rangle$. In this range of scales, $\left\langle R_{x}^{2}\right\rangle$ and $\left\langle R_{y}^{2}\right\rangle$ grow with two different power laws and the local slopes in the inset of Fig. 3 are found to be in 


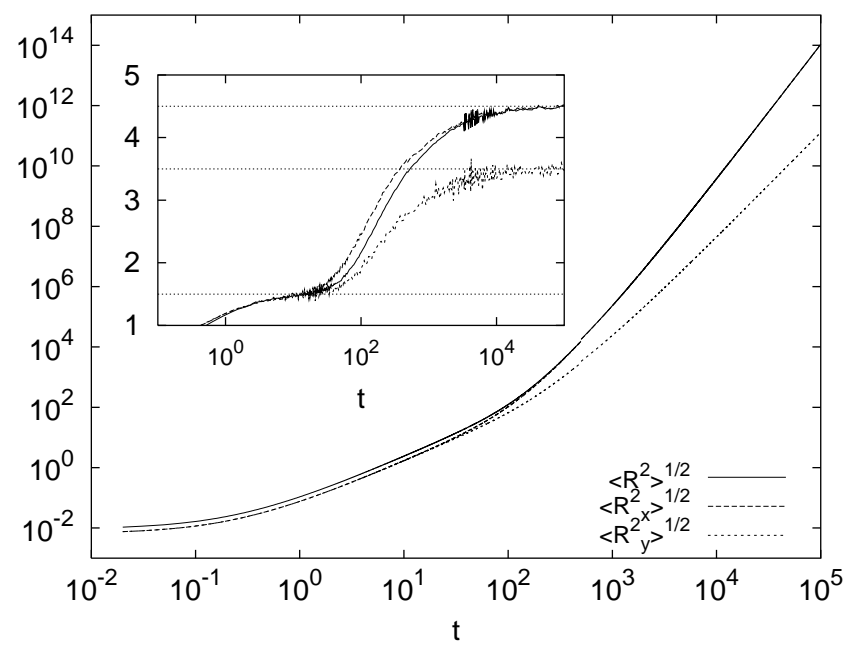

Figure 3. The curves of the Lagrangian separations as a function of time, for a Kraichnan flow with exponent $\xi=4 / 3$. At small times, $\left\langle R_{x}^{2}(t)\right\rangle=\left\langle R_{y}^{2}(t)\right\rangle=\left\langle R^{2}(t)\right\rangle / 2 \sim t^{3}$, as expected according to the Richardson law. At large times, $\left\langle R^{2}(t)\right\rangle \approx\left\langle R_{x}^{2}(t)\right\rangle \sim t^{9}$ and $\left\langle R_{y}^{2}(t)\right\rangle \sim t^{7}$, in agreement with (4.2). The inset displays the local slopes as a function of time. Note the wide crossover region between the two regimes. The numerical integrations of Eqs. (3.5) and (3.6) have been performed following the point-splitting scheme described in Frisch et al. (1998) i.e. with molecular diffusivity $\kappa=0$ and taking the initial separation small but finite.

agreement with (4.2). The predictions (4.2) and the numerical simulations are found to be in agreement for values of $\xi \neq 4 / 3$ as well (data not shown).

An interesting remark is that the Lagrangian numerics evidences a wide crossover region between the two different regimes (see Fig. 3). That is the reason for the very slow convergence of the scalar structure functions and spectra to their asymptotic slopes. In general, detecting the transition between the inertial-convective and the shear regimes demands a very broad range of accessible scales.

We finally consider the case where the shear is random. If its direction is fixed and the time-scale of its intensity is short, the resulting exponent of the scalar spectrum is $-2+\xi / 2$. If both the direction and the intensity of the shear rapidly fluctuate in time, i.e. the shear is a Kraichnan flow with $\xi=2$, isotropy is recovered and the scalar correlation function in the stationary state obeys $(1 / r) \partial_{r}\left(\sigma r^{3}+D r^{1+\xi}+2 \kappa r\right) \partial_{r} C_{2}=-\chi(r)$. The equation is easily solved analytically and the ensuing slopes of the scalar spectra are $-3+\xi$ at small scales (large wavenumbers) and -1 at large scales (small wavenumbers).

\section{Conclusions}

The results presented here illustrate the presence of a new shear regime for a passive scalar transported in turbulent shear flows. The scale of crossover between the classical Obukhov-Corrsin range and the larger scales, where the effects of the shear are felt, is proportional to the ratio between the turbulent and the shear intensities. The range of scales where the shear-regime is observed becomes then parametrically large with increasing the shear rate. In experiments where the latter is fixed, increasing the turbulence intensity has the double effect of decreasing the Kolmogorov scale and increasing the crossover length $r_{c}$. The Obukhov-Corrsin scaling is therefore observed over a broader and broader range of scales. When $r_{c}$ is much larger than the integral scale $L$ of the scalar, the picture 
at scales $\leq L$ becomes essentially shear-free. For $r_{c} \simeq L$, the presence of the shear-range is still relevant due to the crossover effects which induce a scalar spectrum shallower than $-5 / 3$ and anisotropic. Those effects disappear quite slowly with the Reynolds number due to the broad span of scales required for the crossover. Furthermore, we have shown that the scalar spectrum in the shear-region is sensitive to the geometry and the fluctuations of the shear. Experiments with different set-up's, where the shear components have drastically differing properties, might then yield different scalings at asymptotically large scales. All are shallower than $-5 / 3$, though, and therefore inducing crossover effects that tend to flatten out the scalar spectrum. In those conditions where shear fluctuations in intensity and direction are kept controlled, we specifically predict the existence of a $k^{-4 / 3}$ asymptotic regime. The previous picture seems to account for the major points in the experimental observations presented in $\S 2$.

Two issues for future investigation are the following. Theoretically, it is quite likely that the shear will affect the scalar higher-order statistics; the resulting behaviours remain to be clarified. Experimentally, it would be of major interest to have a set-up where the Reynolds number is kept fixed and the shear rate is increased, in well-controlled conditions, so as to enlarge the shear-regime and quantitatively analyze its properties.

The authors acknowledge the hospitality of the Erwin Schrödinger Institute in Vienna. This work has been partially supported by the EU under the contract "Non-Ideal Turbulence" HPRN-CT-2000-00162 and by Cofin 2001 (prot. 2001023848).

\section{Appendix A. Equations for the correlation function and numerical computation}

The anisotropy introduced by the shear can be handled by using polar coordinates, which allow to decompose the correlation function as:

$$
C_{2}(\boldsymbol{r}, t)=C_{2}(r, \phi, t)=F_{0}(r, t)+\sum_{\ell=1}^{\infty} F_{\ell}(r, t) \cos (2 \ell \phi)+\sum_{\ell=1}^{\infty} G_{\ell}(r, t) \sin (2 \ell \phi) .
$$

Here, $\ell$ labels the order of the harmonics. Note that only even harmonics are present for symmetry reasons. Substituting (A 1) into (4.3), one obtains the following set of $1+1$ dimensional partial differential equations:

$$
\begin{aligned}
& \partial_{t} F_{\ell}+\sigma \frac{r}{4}\left[\partial_{r} G_{\ell+1}-\partial_{r} G_{\ell-1}\right]+\frac{\sigma}{2}\left[(\ell+1) G_{\ell+1}-2 \ell G_{\ell}+(\ell-1) G_{\ell-1}\right]= \\
& \frac{1}{r} \partial_{r}\left[2 \kappa r+D r^{1+\xi}\right] \partial_{r} F_{\ell}-\left[2 \kappa+(1+\xi) D r^{\xi}\right] \frac{4 \ell^{2}}{r^{2}} F_{\ell}+\delta_{\ell, 0} \chi \\
& \partial_{t} G_{\ell}-\sigma \frac{r}{4}\left[\partial_{r} F_{\ell+1}-\left(1+\delta_{\ell, 1}\right) \partial_{r} F_{\ell-1}\right]-\frac{\sigma}{2}\left[(\ell+1) F_{\ell+1}-2 \ell F_{\ell}+(\ell-1) F_{\ell-1}\right]= \\
& \frac{1}{r} \partial_{r}\left[2 \kappa r+D r^{1+\xi}\right] \partial_{r} G_{\ell}-\left[2 \kappa+(1+\xi) D r^{\xi}\right] \frac{4 \ell^{2}}{r^{2}} G_{\ell} .
\end{aligned}
$$

Note that the coupling among the harmonics is indeed due to the shear term.

Those equations have been numerically integrated for times long enough to reach a stationary state. The correlation function $C_{2}(r, \phi)$ is then computed at different orientations $\phi$ from $F_{\ell}(r)$ and $G_{\ell}(r)$ by using (A 1). The second-order structure function $S_{2}(r, \phi)=2\left(C_{2}(0)-C_{2}(r, \phi)\right)$ easily follows, as well as the scalar spectrum $E_{\theta}(k, \phi)$, which is obtained by a one-dimensional Fourier transform.

The equations have been solved for various choices of the parameters. In practice, we have fixed $D$ and varied $\sigma$ and $L_{f}$. As for the boundary conditions at the origin, we have 
taken $F_{0}^{\prime}(0)=0, G_{\ell}(0)=0$ for each $\ell$ and $F_{\ell}(0)=0$ for $\ell>0$. The infrared boundary condition has been fixed by setting to zero the values of all the functions $F_{\ell}(r)$ and $G_{\ell}(r)$ beyond $r=L_{b o x}$. The maximum size of the system, $L_{b o x}$, has been taken much larger than the forcing scale $L_{f}$ to properly resolve the anisotropic contributions.

\section{REFERENCES}

BAtChelor, G.K. 1959 Small-scale variation of convected quantities like temperature in turbulent fluid. Part 1. General discussion and the case of small conductivity. J. Fluid Mech. 5, 113-133.

Corrsin, S. 1951 On the spectrum of isotropic temperature field in isotropic turbulence. $J$. Appl. Phys. 22, 469-473.

Falkovich, G., Gawȩdzki, K. \& Vergassola, M. 2001 Particles and fields in fluid turbulence. Rev. Mod. Phys. 73, 913-975.

Frisch, U., Mazzino, A. \& Vergassola, M. 1998 Intermittency in passive scalar advection Phys. Rev. Lett. 80, 5532-5535.

Jayesh, Tong, C. \& Warhaft, Z. 1994 On temperature spectra in grid turbulence. Phys. Fluids 6, 306-312.

Kraichnan, R. H. 1968 Small-scale structure of a scalar field convected by turbulence. Phys. Fluids 11, 945-963.

Levèque, M., A. 1928 Les lois de la transmission de la chaleur par convection. Ann. Mines 13, 201-239.

Mestayer, P. 1982 Local isotropy and anisotropy in a high-Reynolds number turbulent boundary layer. J. Fluid Mech. 125, 475-503.

Miller, P., L. \& Dimotakis, P., E. 1996 Measurement of scalar power spectra in high Schmidt number turbulent jets. J. Fluid Mech. 308, 129-146.

Mydlarski, L. \& WARhaft, Z. 1998 Passive scalar statistics in high-Péclet-number grid turbulence. J. Fluid Mech. 358, 135-175.

Oвuknov, A. M. 1949 The structure of the temperature field in a turbulent flow Izv. Akad. Nauk. SSSR, Ser. Geogr. and Geophys. 13, 58-69.

Siggia, E. D. 1994 High Rayleigh number convection Annu. Rev. Fluid Mech. 26, 137-168.

Sreenivasan, K. R. 1991 On local isotropy of passive scalars in turbulent shear flows. Proc. $R$. Soc. London Ser. A 434, 165-182.

Sreenivasan, K. R. 1996 The passive scalar spectrum and the Obukhov-Corrsin constant. Phys. Fluids 8, 189-196.

Staicu, A. \& van de Water, W. 2003 Small-scale velocity jumps in shear turbulence. Phys. Rev. Lett. 90, 094501-094505.

Villermaux, E., Innocenti, C. \& Duplat, J. 2001 Short circuits in the Corrsin-Obukhov cascade. Phys. Fluids 13, 284-289.

Warhaft, Z. 2000 Passive scalars in turbulent flows. Ann. Rev. Fluids Mech. 32, 203-240.

Yeung, P.K., Xu, S. \& Sreenivasan, K.R. 2002 Schmidt number effects on turbulent transport with uniform mean scalar gradient Phys. Fluids 14, 4178-4191. 